\title{
Encuesta de actitudes y conocimientos médicos en el manejo paliativo hospitalario
}

\author{
Survey on Attitudes and Medical Knowledge at Hospital Palliative Management \\ Inquérito de atitudes e conhecimentos médicos no manejo paliativo hospitalar \\ Juan Rafael López Sánchez MD, MsC'
}

Recibido: 27 de marzo de $2015 \bullet$ Aceptado: 25 de agosto de 2015

Doi: dx.doi.org/10.12804/revsalud14.01.2016.05

Para citar este artículo: López-Sánchez JR. Encuesta de actitudes y conocimientos médicos en el manejo paliativo hospitalario. Rev Cienc Salud. 2016;14(1): 53-61. doi: dx.doi.org/10.12804/revsalud14.01.2016.05

\section{Resumen}

Introducción: El cuidado paliativo se define como el cuidado total activo de los pacientes cuya enfermedad no responde a tratamiento curativo; de esta manera, el presente estudio pretende identificar el nivel de actitudes y conocimientos en cuidados paliativos de un hospital de alto nivel de complejidad. Materiales y métodos: Estudio observacional, de corte transversal, mediante una encuesta validada, auto-suministrada vía web. Se evaluó la perspectiva práctica, grado de conocimiento, grado de comodidad, perspectiva de pertinencia y perspectiva de idoneidad de los encuestados (médicos) en cuidados paliativos; se describieron las características generales, el grado de fiabilidad y los puntajes por ítem y dominio. Resultados: Se encuestaron 145 médicos, la mayoría de sexo masculino, con 0 a 5 años de ejercicio profesional y pertenecientes al área de urgencias (51,03\%), se encontraron apropiados coeficientes de confiabilidad. El nivel global de actitudes y conocimientos de cuidados paliativos fue de 59,09 puntos de 100 posibles, los dominios de menor puntaje fueron las Perspectivas de idoneidad (40,73 puntos) y práctica (47,04 puntos). Conclusiones: La encuesta mostró un nivel de moderado a bajo en actitudes y conocimientos, los puntos más débiles fueron la perspectiva práctica y la idoneidad en la toma de decisiones, deben realizarse capacitaciones dirigidas a establecer pronósticos y mejorar la toma de decisión de remisión al servicio de cuidados paliativos. Los resultados de encuestas de control, posteriores a las capacitaciones, permitirán evaluar las mejoras en los conocimientos y actitudes de los cuidados paliativos.

Palabras clave: Cuidado paliativo, pacientes terminales, enfermedades limitantes para la vida, nivel de conocimientos.

1 Méderi Hospital Universitario Mayor. Universidad del Rosario. Correo electrónico: jrlsmd@hotmail.com 


\section{Abstract}

Introduction: Palliative care is defined as the total active care of patients whose disease is not responsive to curative treatment, therefore this study aims to identify the level of attitudes and knowledge in palliative care hospital with high level of complexity. Materials and methods: Observational, cross-sectional method; using a validated survey. It was self-supplied via web. Perspective practice, knowledge degree, comfort level, perspective relevance and appropriateness of the respondents (doctors) in palliative care were assessed. General characteristics, reliability and scores per item and domain were described. Results: 145 physicians, mostly males, were surveyed, most of them with 0-5 years of professional practice and working in the emergency department $(51.03 \%)$. Appropriate reliability coefficients were found; overall level of attitudes and knowledge of palliative care was 59.09 points out of 100; lowest score domains were the prospects suitability (40.73 points) and practice (47.04 points). Conclusions: The survey showed moderate to low degrees in attitudes and knowledge, the weakest points were practical perspective and expertise in decision-making. Training aimed at establishing prognosis and improving decision making as to referring to palliative care service must be carried out. Survey results of inspection after training will allow an assessment of the improvements in knowledge and attitudes of palliative care.

Keywords: Palliative care, Palliative medicine/education, Hospital medical staff, Interprofessional relations, Evaluation studies, Quality of health care.

\section{Resumo}

Introdução: O cuidado paliativo define-se como o cuidado total ativo dos pacientes cuja doença não responde a tratamento curativo; o presente estudo pretende identificar o nível de atitudes e conhecimentos em cuidados paliativos de um hospital de alto nível de complexidade. Materiais e métodos: estudo observacional, de corte transversal; por meio de um inquérito validado, auto -administrado através da web; avaliou-se a Perspectiva prática; Grau de conhecimento; Grau de comodidade; Perspectiva de pertinência e Perspectiva de idoneidade dos respondentes (médicos) em cuidados paliativos; descreveram-se as características gerais, o grau de fiabilidade e as pontuações por item e domínio. Resultados: inquiriram-se 145 médicos, a maioria de sexo masculino; com 0 a 5 anos de exercício profissional e pertencentes à área de emergências (51,03 \%), encontraram-se apropriados coeficientes de confiabilidade; o nível global de atitudes e conhecimentos de cuidados paliativos foi de 59,09 pontos de 100 possíveis; os domínios de menor pontuação foram as Perspectivas de idoneidade (40,73 pontos) e prática (47,04 pontos). Conclusões: o inquérito mostrou um nível moderado a baixo em atitudes e conhecimentos, os pontos mais fracos foram a perspectiva prática e a idoneidade na tomada de decisões, devem realizar-se capacitações dirigidas a estabelecer prognósticos e melhorar a tomada de decisão de remissão ao serviço de cuidados paliativos; resultados de inquéritos de controle posterior às capacitações permitiram avaliar, as melhoras nos conhecimentos e atitudes dos cuidados paliativos.

Palavras-chave: Cuidado paliativo, pacientes terminais, doenças limitantes para a vida, nível de conhecimento. 


\section{Introducción}

El cuidado paliativo se define como el cuidado total activo de los pacientes cuya enfermedad no responde a tratamiento curativo, donde el control del dolor y de otros síntomas y de problemas psicológicos, sociales y espirituales es primordial (1).

El manejo paliativo debe ser guiado por una evaluación completa de las necesidades biopsicosociales, determinando así el manejo más apropiado del paciente al final de la vida (2).

El manejo interdisciplinario debe lidiar con las incertidumbres y mantener una coordinación efectiva para proveer un mejor cuidado (3).

El hospital universitario mayor Méderi (HUM) en la ciudad de Bogotá, Colombia es un sitio de prácticas de la Universidad de Rosario donde se desarrolla el programa de especialización en dolor y cuidado paliativo. Existe evidencia que soporta que la falta de experiencia y conocimientos en el cuidado paliativo puede ser mejorada al identificar y direccionar cambios simples en la estructura de las instituciones (4).

La Universidad del Rosario, en su Decreto rectoral del año 2004, establece que el norte de las facultades de salud es el alivio del sufrimiento humano, dicho sufrimiento se caracteriza por la sensación que tiene el individuo de sentirse amenazado en la integridad de su persona, por el sentimiento de falta de control sobre dicha amenaza y por el agotamiento de los recursos personales y psicosociales que le permitirían afrontar dicha amenaza. El aforismo de Eric Cassell "Los que sufren no son los cuerpos, son las personas" resume que el sufrimiento es un conflicto de la integridad del ser de la persona y solo puede entenderse y acompañarse desde un modelo integral (4-16).

El presente estudio pretende identificar el nivel de actitudes y conocimientos en cuidados paliativos y expone los puntos débiles para su mejoramiento y ajustes de capacitación en el personal médico del HuM.

\section{Materiales y métodos}

Se realizó un estudio observacional de corte transversal, se aplicó una encuesta validada, auto-suministrado vía web (Encuesta de actitudes y conocimientos en la práctica médica del manejo de pacientes de cuidado paliativo), que cuenta con preguntas cerradas de opción múltiple (preguntas de caracterización de la población y de evaluación del conocimiento y actitudes de los encuestados en cuidados paliativos). La encuesta se encuentra dividida en 42 ítems y 5 dominios así: Perspectiva práctica (9 ítems), grado de conocimiento (10 ítems), grado de comodidad (5 ítems), perspectiva de pertinencia (8 ítems) y perspectiva de idoneidad (10 ítems).

Los registros fueron exportados a una matriz de datos apilados en una hoja de cálculo y, posteriormente, al software estadístico sPSS versión 22.0 para el análisis.

Se recodificaron los ítems a valores en una escala 0 a 100, donde 0 es el peor y 100 el mejor puntaje posible, según la metodología propuesta por RAND, se describieron las características generales de los encuestados, se verificó la confianza en las respuestas por medio del coeficiente alfa de Cronbach y se presentaron los puntajes medios y desviaciones estándar por ítem y dominio.

\section{Resultados}

Se encuestaron 145 médicos del Hum, de un total de 650 existentes en el momento de la aplicación de la encuesta, la mayoría de sexo masculino (52\%), entre los 20 y 30 años de edad $(42,76 \%)$, con 0 a 5 años de ejercicio profesional (45,52 \%). De estos, el 51,03\% se desempeñaban en el área de urgencias, el 40,69 \% manifestaron haber recibido algún tipo de entrenamiento en cuidado paliativo, el 66,9\% tuvo acceso a servicios de cuidados paliativo para remitir a sus pacientes y el 35,17 \% aceptó 
haber remitido más de 10 pacientes en el último año a cuidados paliativos (tabla 1).

Posterior a la recodificación de las categorías en los ítems, según la dirección de las preguntas, se encontraron apropiados coeficientes de confiabilidad para todos los dominios (Alfa de Cronbach de Perspectiva práctica: 0,64; Grado de conocimiento: 0,92; Grado de comodidad: 0,90; Perspectiva de pertinencia: 0,76 y Perspectiva de idoneidad: 0,58). El ítem de menor puntaje fue el 2 de la perspectiva práctica "Dificultad para establecer el pronóstico de pacientes con enfermedades terminales o, en su defecto, enfermedades limitantes para la vida" $(20,12$ puntos); seguido del ítem 37 (en la perspectiva de idoneidad) " ¿Cuándo cree usted que es el momento ideal para derivar un paciente con osteoartritis generalizada al servicio de cuidados paliativos?" con 24,23 puntos (tabla 2).

Tabla 1. Características generales de los encuestados

\begin{tabular}{|c|c|c|c|c|}
\hline \multicolumn{2}{|c|}{ Variable } & Masculino \# $(\%)$ & Femenino \# $(\%)$ & Total \# $(\%)$ \\
\hline \multirow{4}{*}{ Grupos de edad } & $20-30$ & $27(18,62)$ & $35(24,14)$ & $62(42,76)$ \\
\hline & $31-40$ & $12(8,28)$ & $20(13,79)$ & $32(22,07)$ \\
\hline & $41-50$ & $24(16,55)$ & $12(8,28)$ & $36(24,83)$ \\
\hline & $51-60$ & $13(8,97)$ & $2(1,38)$ & $15(10,34)$ \\
\hline \multirow{5}{*}{ Años de ejercicio profesional } & $0-5$ & $30(20,69)$ & $36(24,83)$ & $66(45,52)$ \\
\hline & $6-10$ & $10(6,9)$ & $15(10,34)$ & $25(17,24)$ \\
\hline & $11-15$ & $5(3,45)$ & $9(6,21)$ & $14(9,66)$ \\
\hline & $16-20$ & $15(10,34)$ & $7(4,83)$ & $22(15,17)$ \\
\hline & $>20$ & $16(11,03)$ & $2(1,38)$ & $18(12,41)$ \\
\hline \multirow{6}{*}{ Nivel de formación } & Médico general & $39(26,9)$ & $53(36,55)$ & $92(63,45)$ \\
\hline & Residente de primer año & $0(0)$ & $1(0,69)$ & $1(0,69)$ \\
\hline & Residente de segundo año & $1(0,69)$ & $2(1,38)$ & $3(2,07)$ \\
\hline & Residente de tercer año & $3(2,07)$ & $1(0,69)$ & $4(2,76)$ \\
\hline & Especialista & $24(16,55)$ & $9(6,21)$ & $33(22,76)$ \\
\hline & Supraespecialista & $9(6,21)$ & $3(2,07)$ & $12(8,28)$ \\
\hline \multirow{4}{*}{ Área de desempeño profesional } & Urgencias & $37(25,52)$ & $37(25,52)$ & $74(51,03)$ \\
\hline & Hospitalización & $25(17,24)$ & $23(15,86)$ & $48(33,1)$ \\
\hline & UCI & $4(2,76)$ & $3(2,07)$ & $7(4,83)$ \\
\hline & Consulta externa & $10(6,9)$ & $6(4,14)$ & $16(11,03)$ \\
\hline \multicolumn{2}{|c|}{ Ha recibido algún tipo de conocimiento en cuidado paliativo } & $32(22,07)$ & $27(18,62)$ & $59(40,69)$ \\
\hline \multicolumn{2}{|c|}{$\begin{array}{l}\text { Tiene acceso a servicio de cuidados paliativos para remitir a sus } \\
\text { pacientes }\end{array}$} & $55(37,93)$ & $42(28,97)$ & $97(66,9)$ \\
\hline \multirow{5}{*}{$\begin{array}{l}\text { Número de pacientes derivados } \\
\text { en el último año a cuidados pa- } \\
\text { liativos }\end{array}$} & Sin datos & $22(15,17)$ & $27(18,62)$ & $49(33,79)$ \\
\hline & Ninguno & $3(2,07)$ & $0(0)$ & $3(2,07)$ \\
\hline & entre $1-5$ & $17(11,72)$ & $10(6,9)$ & $27(18,62)$ \\
\hline & entre 6-10 & $9(6,21)$ & $6(4,14)$ & $15(10,34)$ \\
\hline & $>10$ & $25(17,24)$ & $26(17,93)$ & $51(35,17)$ \\
\hline \multicolumn{2}{|l|}{$\mathrm{N}=145$ encuestados } & & & \\
\hline
\end{tabular}


A la evaluación por dominios, los de menor El nivel global en actitudes y conocimientos puntaje fueron las Perspectivas de idoneidad de cuidados paliativos fue de 59,09 puntos de (40,73 puntos) y práctica (47,04 puntos) (tabla 3$).$

Tabla 2. Distribución general de resultados por ítem y escala

\begin{tabular}{|c|c|c|c|c|}
\hline Dominio & Alfa de Cronbach & Ítem & Recuento & Media(DE) \\
\hline \multirow{9}{*}{ Perspectiva práctica } & \multirow{9}{*}{0,64} & Ítem 1 & 81 & $67,16(29,55)$ \\
\hline & & Ítem 2 & 121 & $20,12(27)$ \\
\hline & & Ítem 3 & 121 & $27,93(34,49)$ \\
\hline & & Ítem 4 & 121 & $14,83(21,04)$ \\
\hline & & Ítem 5 & 121 & $57,52(38,09)$ \\
\hline & & Ítem 6 & 121 & $47,11(35,67)$ \\
\hline & & Ítem 7 & 121 & $60(37,86)$ \\
\hline & & Ítem 8 & 121 & $77,19(31,02)$ \\
\hline & & Ítem 9 & 121 & $47,77(39,44)$ \\
\hline \multirow{10}{*}{ Grado de conocimiento } & \multirow{10}{*}{0,92} & Ítem 10 & 112 & $75(29,47)$ \\
\hline & & Ítem 11 & 112 & $78,04(27,89)$ \\
\hline & & Ítem 12 & 112 & $64,82(32,8)$ \\
\hline & & Ítem 13 & 112 & $69,46(33,2)$ \\
\hline & & Ítem 14 & 112 & $74,11(30,92)$ \\
\hline & & Ítem 15 & 112 & $77,68(28,25)$ \\
\hline & & Ítem 16 & 112 & $76,61(27,49)$ \\
\hline & & Ítem 17 & 112 & $75,54(29,5)$ \\
\hline & & Ítem 18 & 112 & $67,32(34,8)$ \\
\hline & & Ítem 19 & 112 & $68,39(35,07)$ \\
\hline \multirow{5}{*}{ Grado de comodidad } & \multirow{5}{*}{0,90} & Ítem 20 & 111 & $64,5(33,86)$ \\
\hline & & Ítem 21 & 111 & $56,22(37,13)$ \\
\hline & & Ítem 22 & 111 & $67,39(33,13)$ \\
\hline & & Ítem 23 & 111 & $58,92(35,35)$ \\
\hline & & Ítem 24 & 111 & $66,49(34,24)$ \\
\hline \multirow{8}{*}{ Perspectiva de pertinencia } & \multirow{8}{*}{$0,76^{*}$} & Ítem 25 & 111 & $87,39(33,35)$ \\
\hline & & Ítem 26 & 108 & $82,22(24,96)$ \\
\hline & & Ítem 27 & 108 & $55,74(34,92)$ \\
\hline & & Ítem 28 & 108 & $57,96(35,33)$ \\
\hline & & Ítem 29 & 108 & $68,52(34,06)$ \\
\hline & & Ítem 30 & 108 & $55,56(34,73)$ \\
\hline & & Ítem 31 & 108 & $32,04(33,93)$ \\
\hline & & Ítem 32 & 108 & $47,96(37,39)$ \\
\hline
\end{tabular}

Continúa 


\begin{tabular}{|c|c|c|c|c|}
\hline Dominio & Alfa de Cronbach & Ítem & Recuento & Media(DE) \\
\hline \multirow{10}{*}{ Perspectiva de idoneidad } & \multirow{10}{*}{$0,58^{*}$} & Ítem 33 & 108 & $44,07(36,35)$ \\
\hline & & Ítem 34 & 104 & $54,04(29,87)$ \\
\hline & & Ítem 35 & 104 & $33,65(32,53)$ \\
\hline & & Ítem 36 & 104 & $33,85(36,45)$ \\
\hline & & Ítem 37 & 104 & $24,23(33,05)$ \\
\hline & & Ítem 38 & 104 & $27,88(32,91)$ \\
\hline & & Ítem 39 & 104 & $46,92(37,37)$ \\
\hline & & Ítem 40 & 104 & $41,92(37,24)$ \\
\hline & & Ítem 41 & 104 & $44,23(37,87)$ \\
\hline & & Ítem 42 & 104 & $56,73(49,78)$ \\
\hline
\end{tabular}

$\mathrm{N}=145$

${ }^{*}$ Alfa de Cronbach estandarizado

Tabla 3. Análisis de fiabilidad, correlación intraclase y estadísticos de escala por dominios (posterior a corrección de direccionalidad de las categorías en los ítems)

\begin{tabular}{lccccccc}
\hline \multirow{2}{*}{ Dominio } & Alfa de & N. ${ }^{\circ}$ de & Media (DE) & \multicolumn{3}{c}{ Correlación intraclase } \\
\cline { 5 - 8 } & Cronbach & ítems & & $\begin{array}{c}\text { Medidas } \\
\text { únicas }\end{array}$ & $\begin{array}{c}\text { Medidas prome- } \\
\text { dio }^{* *}\end{array}$ & Sig \\
\hline Perspectiva práctica & 0,64 & 9 & $47,04(16,68)$ & 0,16 & 0,632 & 0,000 \\
Grado de conocimiento & 0,92 & 10 & $72,7(23,61)$ & 0,531 & 0,919 & 0,000 \\
Grado de comodidad & 0,90 & 5 & $62,7(29,19)$ & 0,631 & 0,895 & 0,000 \\
Perspectiva de pertinencia & $0,76 \uparrow$ & 8 & $61,11(20,78)$ & 0,296 & 0,771 & 0,000 \\
Perspectiva de idoneidad & $0,58 \uparrow$ & 10 & $40,73(16,13)$ & 0,103 & 0,535 & 0,000 \\
Total & 0,76 & 42 & $59,09(10,23)$ & 0,07 & 0,759 & 0,000 \\
\hline
\end{tabular}

* Coeficientes de correlaciones entre clases del tipo C utilizando una definición de coherencia. La varianza de medida intermedia se excluye de la varianza del denominador.

** Esta estimación se calcula suponiendo que el efecto de interacción está ausente, porque de lo contrario no se puede estimar.

ฯ Alfa de Cronbach estandarizado

\section{Discusión}

La etapa terminal de una enfermedad es un proceso difícil de afrontar, no solo se encuentra presente en enfermedades oncológicas, también hace parte de enfermedades no oncológicas como lo evidencia de MacNamara, en patologías como la Insuficiencia Cardiaca, Insuficiencia Renal, Insuficiencia Hepática, Enfermedad
Pulmonar Obstructiva Crónica, Esclerosis Lateral Amiotrófica, Enfermedades de la Motoneurona, Enfermedad de Parkinson, Corea de Huntington, Alzheimer y sIDA (6). Esto genera un impacto emocional tanto para el paciente como para la familia y el equipo tratante.

Otorgar calidad de vida al paciente con enfermedad terminal para que muera con 
serenidad, dignidad y paz total implica que la muerte del paciente se produzca en las mejores condiciones.

Se aplicó una encuesta al personal médico de un hospital de alta complejidad para la medición de actitudes y conocimientos en cuidados paliativos, se definieron las características generales de los encuestados, se verificó la confiabilidad de los resultados (por coeficiente alfa de Cronbach) y se expusieron los puntos débiles para su mejoramiento.

La atención paliativa se centra en el control del dolor y otros síntomas, lo que evita el sufrimiento del paciente y de su familia, por tanto, dicho cuidado o atención engloba diversas esferas, incluyendo el manejo de dolor total. Esta es una situación, en principio, relacionada con el cáncer avanzado que también existe en el SIDA y en la fase final de otras enfermedades (7).

El personal debería tener un espíritu humanístico y estar sensibilizado, concientizado, familiarizado y capacitado para poder otorgar estos cuidados especializados $(8,9)$.

Según los resultados, existe dificultad en los médicos para establecer pronóstico en los pacientes con enfermedades terminales o limitantes para la vida, esto podría motivar a los familiares de los pacientes a solicitar intervenciones de sostén no indicadas; independiente de que actúen o no terapéuticamente sobre la enfermedad de base o el proceso biológico que amenaza la vida del paciente (10). Las situaciones que requieren cuidados paliativos varían y son difíciles de predecir (11). La aproximación paliativa debe basarse en las necesidades del paciente y de su familia, más que en el pronóstico de su enfermedad; por ello, el manejo deberá ser estandarizado y permitir la muerte sin mayores impedimentos, brindando el apoyo necesario para que sea lo más tranquila posible (12).

Otro de los puntos débiles detectados en la encuesta fue el desconocimiento de la indica- ción apropiada para derivar pacientes a cuidados paliativos, en particular en caso de osteoartritis, lo que sugiere el imperativo desarrollo de capacitaciones en estos aspectos. La carga sintomatológica experimentada por estos pacientes es comparable a la que sufren los pacientes con cáncer al final de la vida. Sin embargo, cuando se comparan los cuidados prestados a pacientes con una enfermedad oncológica terminal con los dispensados a pacientes con insuficiencias orgánicas en fase avanzada, los resultados son siempre mejores en el primer grupo; probablemente secundario a la dificultad para realizar un pronóstico vital en este tipo de pacientes (13).

Las encuestas fueron respondidas por personal voluntario, quienes, de alguna forma, podrían verse más motivados a responder en la medida que considerasen mayor su conocimiento del área. Este sesgo es habitual y no controlado en las encuestas voluntarias, además hubo 24 encuestas que no fueron completadas en totalidad y cuyos datos no se toman en cuenta para su análisis.

Como fortaleza se señala que es una primera aproximación al conocimiento y actitudes al cuidado paliativo en un hospital colombiano. Los resultados del presente estudio serán de utilidad para el diseño de intervenciones sanitarias encaminadas a mejorar las actitudes y conocimientos en los cuidados paliativos (14).

\section{Conclusiones}

Se identificó un nivel de moderado a bajo en las actitudes y conocimientos en cuidados paliativos de los médicos evaluados. Los puntos más débiles para mejoramiento son la perspectiva práctica y de idoneidad en la toma de decisiones, deben realizarse capacitaciones dirigidas a establecer pronósticos y mejorar la toma de decisión de remisión al servicio de cuidados paliativos. 
Los ajustes de capacitación del personal médico deberán también tener conceptos fundamentales de medicina geriátrica y bioética, además de medicina paliativa.

Los resultados de encuestas de control, posterior a las capacitaciones, permitirán evaluar, de forma objetiva, las mejoras en los conocimientos y actitudes de los cuidados paliativos por los tratantes.
Los cuidados paliativos son un tipo especial de cuidados intensivos que requieren un sentido de urgencia, atención al detalle, deben centrarse en las necesidades del paciente y de su familia y deberán ser biológicamente adecuados con poca tecnología y mucho tacto.

\section{Referencias}

1. Murray SA, Kendall M, Boyd K, Sheikh A. Illness trajectories and palliative care. BMJ 2005;330(7498):1007-11.

2. Ferrell B, Levy MH, Paice J. Managing pain from advanced cancer in the palliative care setting. Clin J Oncol Nurs. 2008;12(4):575-81.

3. Oishi A, Murtagh FEM. The challenges of uncertainty and interprofessional collaboration in palliative care for non-cancer patients in the community: a systematic review of views from patients, carers and health-care professionals. Palliat Med. 2014; 28(9):1081-98.

4. Browne S, Macdonald S, May CR, Macleod U, Mair FS. Patient, carer and professional perspectives on barriers and facilitators to quality care in advanced heart failure. PLoS One. 2014; 9(3):e93288.

5. Jr JW, Sherbourne C. The MOS 36-item short-form health survey (SF-36): I. Conceptual framework and item selection. Med Care. 1992; 30:473-83.

6. Ministerio de Sanidad, Política Social e Igualdad. Estrategia en Cuidados Paliativos del Sistema Nacional de Salud actualización 2010-2014. Sanidad 2011. [internet]. 2011 [citado 2015 mar 25]. Disponible en: http://www.mspsi.gob.es/organizacion/sns/planCalidadSNS/docs/paliativos/cuidadospaliativos.pdf

7. Saunders CM. Cuidados de la Enfermedad Maligna Terminal. Cataluña: Universidad Central de Cataluña, Ed Salvat; 1980.

8. Kübler-Ross E. La Rueda de La Vida Brito A Trad. México ediciones; México D.F.: 2006 [citado 2015 ene 11]. Disponible en: https://books.google.com.co/books? $\mathrm{id}=4 \mathrm{M} 4 \mathrm{PPAAACAAJ} \& \mathrm{dq}=\mathrm{K} \% \mathrm{C} 3 \% \mathrm{BCb}$ ler-Ross+E,+Brito+A.+La+Rueda+de+La+Vida.\&hl=es\&sa=X\&redir_esc=y A

9. Sancho MG. Morir con dignidad. [internet]. Madrid: Arán Ediciones; 2005 [citado 2015 ene 11]. Disponible en: https://books.google.com/books?id=vSLE4cGPJbUC\&pgis=1

10. Ramos A, Ramos L. Conocimiento sobre cuidados paliativos de personal que labora en el hospital general de Tula de Allende, Hidalgo en 2013 [tesis de diplomado]. [México]: Asociación Mexicana de Tanatología, A. C.; [internet]. 2013 [citado 2015 ene 11]; Disponible en: http://www.tanatologia-amtac. com/descargas/tesinas/157 Conocimiento.pdf

11. Flórez SP, Tovar MB, León MX, Villegas K, Villamizar D del P, Granados CE. Caracterización del conocimiento en cuidado paliativo pediátrico y percepción de barreras por parte de los pediatras y residentes de pediatría. MEDIPAL. 2015;22:127-35.

12. Bouza-Álvarez C. Medidas de soporte vital en un paciente en estado vegetativo persistente. Med Intensiva. 2004; 28(3):156-61. 
13. López R, Nervi F, Pascual-López A, Centeno-Cortés C, Gómez-Sancho M, Naval-Vicuña M. Manual de medicina paliativa. Med. [internet] 2009. Disponible en: http://www.cuidadospaliativos.org/archives/ medicinafinal.pdf

14. Kosinski M, Keller SD, Ware JE, Hatoum HT, Kong SX. The SF-36 Health Survey as a generic outcome measure in clinical trials of patients with osteoarthritis and rheumatoid arthritis: relative validity of scales in relation to clinical measures of arthritis severity. Med Care. 1999;37(Suppl 5):MS23-39.

15. Nichol MB, Sengupta N, Globe DR. Evaluating quality-adjusted life years: estimation of the health utility index (HUI2) from the SF-36. Med Decis Making. 2001;21(2):105-12.

16. Benito E, Maté J, López P. Estrategias para la detección, exploración y atención del sufrimiento en el paciente. FMC 2011;18 (7):392-400. 